\title{
Effect of Vitamin C Supplementation on Electrolyte Concentrations in Blood and Urine of Water Deprived Goats
}

\author{
Sumeet Kour $^{1 *}$, Jonali Devi ${ }^{1}$, Kamal Sarma ${ }^{2}$, Dibyendu Chakraborty ${ }^{3}$, \\ Aafreen Wahid Ganai ${ }^{4}$, Pallavi Khajuria ${ }^{1}$ and Sourab Dua ${ }^{5}$ \\ ${ }^{1}$ Division of Veterinary Physiology and Biochemistry, FVSc\& AH, SKUAST-J, \\ R S Pura, 181102, Jammu, India \\ ${ }^{2}$ Division of Veterinary Anatomy, FVSc\& AH, SKUAST-J, R S Pura, 181102, Jammu, India \\ ${ }^{3}$ Division of Animal Genetics \& Breeding, FVSc\& AH, SKUAST-J, R S Pura, \\ 181102, Jammu, India \\ ${ }^{4}$ Division of Veterinary Public Health, FVSc\& AH, SKUAST-J, R S Pura, 181102, \\ Jammu, India \\ ${ }^{5}$ Division of Livestock Products and Technology, FVSc\& AH, SKUAST-J, R S Pura, 181102, \\ Jammu, India \\ *Corresponding author
}

\section{A B S T R A C T}

The present investigation was aimed to study the effect of vitamin $\mathrm{C}$ on electrolyte concentrations in blood and urine in water deprived goats during summer and winter seasons. Eighteen adult male goats were selected for the experiment during both seasons.In each season, there were two treatment groups: T1 and T2 group. In T1 group, water was restricted and subdivided into three experimental periods: in period 1 (day 1 to 7 ), goats were adapted to the

\section{Keywords}

Goats, Vitamin C, Electrolytes, Water restriction, Summer, Winter

\section{Article Info}

Accepted:

20 November 2019

Available Online:

10 December 2019 water restriction regime by limiting access to water gradually from 15 to 3 hour per day. During the second period of the experiment (day 8 to 14), animals had access to water for 3 hours/day. In the final period of the experiment (day 15 to 22), animals had access to water only every second day for 6 hours. In T2 group, the same protocol as mentioned above was followed with addition of vitamin $\mathrm{C}$ supplementation $(180 \mathrm{mg} / \mathrm{kg}$ body weight/animal/day). In the control group (both season), water was offered ad libitum ( 24 hours) throughout the experimental period. Blood and urine samples were collected and concentrations of different electrolytes were estimated. In T1 group, serum and urine sodium, potassium and chloride levels showed increasing trend $(\mathrm{P}<0.05)$ in both seasons.Urine sodium and chloride concentrations showed increasing trend up to day 22 in both seasons in $\mathrm{T} 2$ group; whereas, potassium concentration showed similar trend from day 7 during winter season, but during summer concentration increased up to 7 day, then decreased. Significantly lower $(\mathrm{P}<0.05)$ serum and urine electrolyte values were observed in vitamin $\mathrm{C}$ supplemented group as compared to water deprived group in last days (14 and 22 day) of experiment. The overall summer value of serum sodium and chloride were higher as compared to overall winter values in all the groups; whereas, overall summer value of potassium was higher only in control group as compared to winter season.Overall summer value of urine sodium was higher in all groups, significant variation found in T2 group. Overall potassium concentrations in urine was significantly higher $(\mathrm{P}<0.05)$ in T1 in summer. Summer chloride concentrations in urine were higher in treatment groups; whereas, lower in control.Water deprivation had negative impact on electrolyte balance, which can be ameoliorated by supplementation of ascorbic acid at the rate of @ $180 \mathrm{mg} / \mathrm{kg}$ b.wt./day. 


\section{Introduction}

Livestock undergo various kinds of stress such as physical, nutritional, chemical, psychological and thermal. Among all, thermal stress is the most concerning now a day in the ever changing climatic scenario. Thermal stress redistributes the body resources including protein and energy at the cost of decreased growth, reproduction, production and health. Climate change is expected to account for about 20 percent of the global increase in water scarcity this century. The Intergovernmental Panel on Climate Change (IPCC) predicts that global warming will alter precipitation patterns around the world, melt mountain glaciers and worsen the extremes of droughts and floods. A study by the UK Meteorological Office predicts that if measures are not taken to mitigate climate change, severe droughts now occurring once every 50 years could occur every other year by 2050 . Meteorological data of Jammu region during last 25 years indicated that the environmental temperature is ranged between $4^{\circ} \mathrm{C}$ in winter to $48^{\circ} \mathrm{C}$ at summer season with relative humidity ranging from 18 to $91 \%$. The areas of $\mathrm{J} \& \mathrm{~K}$ are characterized by water scarcity and fluctuating precipitation, under the effect of global warming and unpredictable weather, rainfall is becoming even more irregular and water availability become more limited. Annual rainfall in Jammu province in the year 2015 was $1909.7 \mathrm{mms}$ with an average of 914.4 $\mathrm{mm} /$ year. Along the water accessibility, feed and other resources will be markedly affected by climate change. These wide ranges of environmental temperature alter the physiological status and lead to stress condition in animals.

Water is one of the most important nutrients, being the most abundant molecule in all living cells (NRC, 2007); it is involved virtually in all physiological functions of the animal.
Water restriction affects animal's physiological homeostasis which included electrolyte imbalance, changes of osmolarity and blood $\mathrm{pH}$, hormonal changes, haematobiochemical changes etc. Decreased feed intake becomes an additional burden on the water stressed animal. Blood metabolites are considered to be the most accurate measure for assessing the physiological status of goats compared to other measures used such as body condition scoring and body weight gain (Madziga et al., 2013). Animal producer and researchers have looked for ways to alleviate the negative effects of common stressors. Stress alleviation strategies are numerous, and their availability to producers depend on the access to water and energy, the price they are able to pay and the adopted farming system. These strategies vary from simple on farm practices such as modifying the feeding pattern such as feeding of vitamin $\mathrm{C}$, electrolyte therapy, etc. Very few studies have been undertaken to see the effect of vitamin $\mathrm{C}$ in water stressed pigs, Japanese quails, rabbits and broilers. Vitamin $\mathrm{C}$ is known for the function as an antioxidant mainly it act as a free radical scavenger in numerous cellular oxidation processes. The results obtained on the effect of vitamin $\mathrm{C}$ on blood electrolytes are not very clear and need more elaborated work. The present study is aimed at examining in detail the changes in electrolyte balance in water restricted goats during extreme climates of Jammu and the likely beneficial effects, if any, produced by vitamin $\mathrm{C}$ supplementation.

\section{Materials and Methods}

The study was conducted in the Division of Veterinary Physiology \& Biochemistry, Faculty of Veterinary Sciences and Animal Husbandry, Sher-e-Kashmir University of Agricultural Sciences and TechnologyJammu, R. S. Pura, Jammu, J\&K, India. Eighteen adult male goats of 2-3 years of age were selected and kept in the Divisional Farm 
of Animal Nutrition, F.V.Sc. \& A.H., SKUAST-Jammu. All animals were provided with standard concentrate ration and maintained under standard managemental conditions during the experimental period. The experiment was conducted during both summer and winter seasons. In the control group ( $n=6$, for each season), water was offered ad libitum (24 hours) throughout the experimental period. In each season, there was two (2) treatment groups (T1 and T2) consisting of 6 animals in each group. In first treatment group (T1), water was restricted and subdivided into three experimental periods: in period 1 (experimental days 1 to 7), animals were adapted to the water restriction regime by limiting access to water gradually from 15 to 3 hours per day. During the second period of the experiment (experimental days 8 to 14), animals of the treatment groups had access to water for 3 hours/day. In the final period of the experiment (experimental days 15 to 22), animals had access to water only every second day for 6 hours. In second treatment group (T2), the same protocol as mentioned above was followed with addition of vitamin $\mathrm{C}$ supplementation at a dose rate of $180 \mathrm{mg} / \mathrm{kg}$ body weight/animal/day. On 1, 7, 14 and 22 day of experiment, $5 \mathrm{ml}$ of blood was collected by venipuncture aseptically from jugular vein of the goats from each group. Then blood sample was allowed to clot and serum was separated and collected in storage vials. Urine was collected by free catch/voided method from each animal on the same days i.e. 1, 7, 14and 22 day of experiment. Sodium and chloride was analyzed by colorimetric method based on modified Maruna and Trinders method and potassium by turbidimetric method in serum and urine using analytical kits manufactured by Agappe diagnostics Ltd., Ernakulam (Kerala), India. The unit of expression for serum $\mathrm{Na}, \mathrm{K}$ and $\mathrm{Cl}$ was as $\mathrm{mEq} / \mathrm{L}$, for urine $\mathrm{Na}$ and $\mathrm{K}$ was $\mathrm{mmol} /$ day and for urine chloride was $\mathrm{g} / 24 \mathrm{hr}$. For all the observed data in the present experiment, the standard statistical procedures have been followed (Snedecor and Cochran, 2004). The data were presented by showing mean and standard error. The significant differences of values for different parameters studied were assessed by the test of two-way analysis of variance depending upon the data. The significant values of different groups and weeks were calculated by Tukey's test. All the above calculations were carried out using SPSS software version 16.0.

\section{Results and Discussion}

\section{Serum electrolytes (sodium, potassium and chloride)}

Analyzing osmolarity is a good approach for monitoring the hydration status. It has been proved that osmolality and electrolyte levels are largely affected by water deprivation. The present study (Table 1) revealed that the serum sodium, potassium and chloride concentrations showed an increasing trend from day 1 to 22 day of water deprivation during both seasons. Under dehydration, in order to preserve homeostasis (Silanikove, 1992; Abdoun et al., 2010; Qinisa et al., 2011) renal water and sodium retention is increased to preserve the blood sodium levels. Increase renal retention of sodium is a physiological response to water restriction in different small ruminant breeds, which allows the maintenance of sodium balance in the body. Ashour and Benlemlih (2001) attributed the increased renal retention to the influence of aldosterone, whereas, Mckinley et al., (2000) added the effect of vasopressin secretion. Dehydration causes an increase in vasopressin levels in both lactating (Maltz et al., 1984; Hossaini-Hilali et al., 1994 and Mengistu et al., 2007) and non-lactating animals (Maltz et al., 1984). Igbokwe (1997) reported increase in serum sodium, potassium and chloride concentration in water deprived goats, sheep and cattle deprived for 4 days which is fully in 
agreement with the study in Osmanabadi goats (Zetingrao, 2003). Rajkhowa and Hazarika (2000) recorded the serum sodium increased significantly on 48 hours onwards with the maximum values recorded (198.21 \pm 2.25 $\mathrm{mEq} / \mathrm{l})$ at 96 hours of water deprivation than the values as zero hours of water deprivation $(145 \pm 0.06 \mathrm{mEq} / 10)$; maximum values of serum chloride on 48 hours onwards with maximum values $(124.23 \pm 2.21 \mathrm{mEq} / \mathrm{l})$ at 96 hours of water deprivation than the values at zero hours of water deprivation $(98.28 \pm 1.25$ $\mathrm{mEq} / \mathrm{l})$. They also reported slight increase in serum potassium from $4.00 \pm 0.07$ to $4.22 \pm 1.14$ $\mathrm{mEq} / \mathrm{l}$ during 96 hours of water deprivation. Samara et al., (2012) observed that dehydration in goats significantly $(\mathrm{P}<0.05)$ increased serum concentrations of sodium and potassium and therefore serum osmolality in goat and camel was increased with parallel increased in their serum sodium concentration during dehydration period; but with smaller percentage changes in camel $(6 \%)$ compared with goat (69\%). Jaber et al., (2004) recorded a decrease in serum potassium concentration in Awassi sheep when subjected to intermittent watering once every 4 days. Aradi goats also exhibited a decrease in serum potassium concentration after 2 days and more after 4 days of water deprivation (El-Nouty et al., 1990).

On the other hand, Cole (2000) and Bashier et al., (2009) did not find any variation in sheep serum sodium and potassium due to water deprivation. The present study (Table 1) revealed a decrease of sodium level from day 7 in summer and sodium, potassium and chloride concentrations from day 14 in vitamin supplemented group in both seasons.

Ghanem et al., (2008) reported that vitamin C administration alleviated the effect of water restriction as reflected in our study which attributed the role of vitamin $\mathrm{C}$ in norepinephrine formation, which affects the kidney function and therefore water and electrolyte dynamics. Karnib (2009) and Hanna (2006) observed increased osmolality in water stressed vitamin $\mathrm{C}$ supplemented Awassi sheep, but the mechanism of such phenomenon is still not well understood. On the other hand, others (Varady et al., 2007) said there was no difference between sodium and chloride ion levels between supplemented and non-supplemented water restricted ewes. The overall summer value of sodium and chloride were higher as compared to overall winter values in all the groups (Table 1); whereas overall summer value of potassium was higher only in control group as compared to winter season.

The increased serum sodium and potassium concentrations observed during the study might be due to dehydration. However, it is also depending on the amount of water intake (Al-Haidary et al., 2012; Wojtas et al., 2014). Rathwa et al., (2017) observed that the level of serum sodium and potassium was increased significantly $(\mathrm{P}<0.05)$ during summer season. Boyd (1981) reported that during thermal stress along with water deprivation for 3-9 days, serum inorganic potassium was increased (7-12\%) in horse, donkey and cattle; whereas no changes were observed in sheep and camel.

\section{Urine electrolytes (sodium, potassium and chloride)}

The present study (Table 2) revealed increasing trend in concentrations of sodium, potassium and chloride in urine in water deprived (T1) group from day 1 to 22 in both seasons. Basheir et al., (2009) observed that water deprivation caused a significant increase in sodium $(\mathrm{P}<0.05)$ ion concentration; whereas, water deprivation did not affect the urinary concentration of potassium ion. Similar finding was also observed by Leng et al., (1987) in sheep. 
Table.1 Mean \pm SE values of serum sodium, potassium and chloride level (mEq/L) in control and treatment groups of goats during summer and winter seasons

\begin{tabular}{|c|c|c|c|c|c|c|c|c|c|c|}
\hline \multirow{2}{*}{\begin{tabular}{|c|} 
Season \\
Days \\
Group
\end{tabular}} & \multicolumn{5}{|c|}{ Summer } & \multicolumn{5}{|c|}{ Winter } \\
\hline & 1 & 7 & 14 & 22 & Total & 1 & 7 & 14 & 22 & Total \\
\hline \multicolumn{11}{|l|}{ Sodium } \\
\hline Control & $\begin{array}{c}119.91 \\
\pm 0.62\end{array}$ & $\begin{array}{c}120.05^{\mathrm{A}} \\
\pm 0.87\end{array}$ & $\begin{array}{c}121.12^{\mathrm{A}} \\
\pm 0.89\end{array}$ & $\begin{array}{c}120.89^{\mathrm{A}} \\
\pm 0.74\end{array}$ & $\begin{array}{c}120.29^{\mathrm{Aq}} \\
\pm 0.40\end{array}$ & $\begin{array}{c}116.45^{\mathrm{ab}} \\
\pm 1.03\end{array}$ & $\begin{array}{c}117.75^{\text {Aab }} \\
\pm 0.74\end{array}$ & $\begin{array}{c}115.94^{\mathrm{Aa}} \\
\pm 0.37\end{array}$ & $\begin{array}{c}119.13^{\mathrm{Ab}} \\
\pm 0.55\end{array}$ & $\begin{array}{c}117.31^{\mathrm{Ap}} \\
\pm 0.42\end{array}$ \\
\hline T1 & $\begin{array}{c}119.55^{\mathrm{a}} \\
\pm 0.66\end{array}$ & $\begin{array}{c}125.10^{\mathrm{Bb}} \\
\pm 0.75\end{array}$ & $\begin{array}{c}135.04^{\mathrm{Bc}} \\
\pm 1.11\end{array}$ & $\begin{array}{c}142.15^{\mathrm{Bd}} \\
\pm 1.31\end{array}$ & $\begin{array}{c}130.46^{\mathrm{B}} \\
\pm 1.88\end{array}$ & $\begin{array}{c}119.10^{\mathrm{a}} \\
\pm 0.85\end{array}$ & $\begin{array}{c}124.70^{\mathrm{Bb}} \\
\pm 1.32\end{array}$ & $\begin{array}{c}132.95^{\mathrm{Cc}} \\
\pm 0.98\end{array}$ & $\begin{array}{c}140.78^{\mathrm{Bd}} \\
\pm 0.86\end{array}$ & $\begin{array}{c}129.38^{\mathrm{B}} \\
\pm 1.78\end{array}$ \\
\hline $\mathbf{T} 2$ & $\begin{array}{c}121.01^{\mathrm{a}} \\
\pm 1.00\end{array}$ & $\begin{array}{c}124.77^{\mathrm{Bb}} \\
\pm 0.72\end{array}$ & $\begin{array}{c}121.08^{\mathrm{Aa}} \\
\pm 0.84\end{array}$ & $\begin{array}{c}120.06^{\mathrm{Aa}} \\
\pm 0.74\end{array}$ & $\begin{array}{c}121.73^{\mathrm{A}} \\
\pm 0.54\end{array}$ & $\begin{array}{c}115.81^{\mathrm{a}} \\
\pm 1.33\end{array}$ & $\begin{array}{c}120.58^{\mathrm{ABb}} \\
\pm 1.35\end{array}$ & $\begin{array}{c}122.73^{\mathrm{Bb}} \\
\pm 0.99\end{array}$ & $\begin{array}{c}120.85^{\mathrm{Ab}} \\
\pm 0.92\end{array}$ & $\begin{array}{c}119.99^{\mathrm{A}} \\
\pm 0.76\end{array}$ \\
\hline \multicolumn{11}{|l|}{ Potassium } \\
\hline Control & $\begin{array}{c}4.01 \\
\pm 0.23\end{array}$ & $\begin{array}{c}4.50 \\
\pm 0.24\end{array}$ & $\begin{array}{l}4.15^{\mathrm{A}} \\
\pm 0.22\end{array}$ & $\begin{array}{l}4.43^{\mathrm{A}} \\
\pm 0.17\end{array}$ & $\begin{array}{l}4.27^{\mathrm{A}} \\
\pm 0.11\end{array}$ & $\begin{array}{c}4.25 \\
\pm 0.16\end{array}$ & $\begin{array}{l}4.01^{\mathrm{A}} \\
\pm 0.14\end{array}$ & $\begin{array}{l}4.08^{\mathrm{A}} \\
\pm 0.16\end{array}$ & $\begin{array}{l}4.08^{\mathrm{A}} \\
\pm 0.19\end{array}$ & $\begin{array}{l}4.19^{\mathrm{A}} \\
\pm 0.08\end{array}$ \\
\hline T1 & $\begin{array}{l}4.27^{\mathrm{a}} \\
\pm 0.15\end{array}$ & $\begin{array}{l}4.81^{\mathrm{ab}} \\
\pm 0.19\end{array}$ & $\begin{array}{l}5.31^{\mathrm{Bb}} \\
\pm 0.12\end{array}$ & $\begin{array}{l}6.26^{\mathrm{Bc}} \\
\pm 0.17\end{array}$ & $\begin{array}{l}5.16^{\mathrm{B}} \\
\pm 0.17\end{array}$ & $\begin{array}{l}4.34^{\mathrm{a}} \\
\pm 0.01\end{array}$ & $\begin{array}{l}4.94^{\mathrm{Bb}} \\
\pm 0.17\end{array}$ & $\begin{array}{l}5.43^{\mathrm{Bb}} \\
\pm 0.11\end{array}$ & $\begin{array}{l}6.21^{\mathrm{Cc}} \\
\pm 0.11\end{array}$ & $\begin{array}{l}5.23^{\mathrm{C}} \\
\pm 0.16\end{array}$ \\
\hline $\mathbf{T} 2$ & $\begin{array}{c}4.54 \\
\pm 0.28\end{array}$ & $\begin{array}{c}4.91 \\
\pm 0.26\end{array}$ & $\begin{array}{l}5.17^{\mathrm{B}} \\
\pm 0.25\end{array}$ & $\begin{array}{l}4.80^{\mathrm{A}} \\
\pm 0.23\end{array}$ & $\begin{array}{l}4.85^{\mathrm{B}} \\
\pm 0.13\end{array}$ & $\begin{array}{c}4.72 \\
\pm 0.12\end{array}$ & $\begin{array}{l}4.91^{\mathrm{B}} \\
\pm 0.11\end{array}$ & $\begin{array}{l}5.05^{\mathrm{B}} \\
\pm 0.09\end{array}$ & $\begin{array}{l}4.75^{\mathrm{B}} \\
\pm 0.13\end{array}$ & $\begin{array}{l}4.86^{\mathrm{B}} \\
\pm 0.06\end{array}$ \\
\hline \multicolumn{11}{|l|}{ Chloride } \\
\hline Control & $\begin{array}{c}100.46^{\mathrm{a}} \\
\pm 0.86\end{array}$ & $\begin{array}{c}104.01^{\mathrm{Ab}} \\
\pm 0.60\end{array}$ & $\begin{array}{c}102.14^{\mathrm{Aab}} \\
\pm 0.46\end{array}$ & $\begin{array}{c}101.09^{\mathrm{Aa}} \\
\pm 0.45\end{array}$ & $\begin{array}{c}101.92^{\mathrm{Aq}} \\
\pm 0.40\end{array}$ & $\begin{array}{c}100.21 \\
\pm 0.68\end{array}$ & $\begin{array}{c}98.69^{\mathrm{A}} \\
\pm 0.61\end{array}$ & $\begin{array}{c}101.15^{\mathrm{A}} \\
\pm 0.58\end{array}$ & $\begin{array}{c}99.96^{\mathrm{A}} \\
\pm 0.61\end{array}$ & $\begin{array}{c}100.00^{\mathrm{Ap}} \\
\pm 0.34\end{array}$ \\
\hline T1 & $\begin{array}{c}103.17^{\mathrm{a}} \\
\pm 0.85\end{array}$ & $\begin{array}{c}112.26^{\mathrm{Cb}} \\
\pm 0.26\end{array}$ & $\begin{array}{c}120.34^{\mathrm{Cc}} \\
\pm 0.44\end{array}$ & $\begin{array}{c}128.00^{\mathrm{Cd}} \\
\pm 0.76\end{array}$ & $\begin{array}{c}115.94^{\mathrm{Cq}} \\
\pm 1.95\end{array}$ & $\begin{array}{c}99.93^{\mathrm{a}} \\
\pm 0.61\end{array}$ & $\begin{array}{c}107.08^{\mathrm{Bb}} \\
\pm 0.69\end{array}$ & $\begin{array}{c}115.15^{\mathrm{Cc}} \\
\pm 0.98\end{array}$ & $\begin{array}{c}120.32^{\mathrm{Cd}} \\
\pm 0.92\end{array}$ & $\begin{array}{c}110.62^{\mathrm{Cp}} \\
\pm 1.66\end{array}$ \\
\hline $\mathbf{T} 2$ & $\begin{array}{c}101.86^{\mathrm{a}} \\
\pm 0.80\end{array}$ & $\begin{array}{c}108.89^{\mathrm{Bb}} \\
\pm 1.10\end{array}$ & $\begin{array}{c}109.43^{\mathrm{Bb}} \\
\pm 0.68\end{array}$ & $\begin{array}{c}104.25^{\mathrm{Ba}} \\
\pm 0.30\end{array}$ & $\begin{array}{c}106.11^{\mathrm{B}} \\
\pm 0.75\end{array}$ & $\begin{array}{c}101.25^{\mathrm{a}} \\
\pm 0.60\end{array}$ & $\begin{array}{c}107.07^{\mathrm{Bb}} \\
\pm 0.62\end{array}$ & $\begin{array}{c}108.43^{\mathrm{Bb}} \\
\pm 0.45\end{array}$ & $\begin{array}{c}102.77^{\mathrm{Ba}} \\
\pm 0.43\end{array}$ & $\begin{array}{c}104.88^{\mathrm{B}} \\
\pm 0.66\end{array}$ \\
\hline
\end{tabular}

$\mathrm{a}, \mathrm{b}, \mathrm{c}, \mathrm{d}$ describe significant differences within groups $(\mathrm{P}<0.05)$

A, B, C describe significant differences between groups $(\mathrm{P}<0.05)$

$\mathrm{p}$, q describe significant differences between seasons $(\mathrm{P}<0.05)$ 
Table.2 Mean \pm SE values of urine sodium (mmol/d), potassium (mmol/day) and chloride (gm/24hr) in control and treatment groups of goats during summer and winter seasons

\begin{tabular}{|c|c|c|c|c|c|c|c|c|c|c|}
\hline Season & \multicolumn{5}{|c|}{ Summer } & \multicolumn{5}{|c|}{ Winter } \\
\hline $\begin{array}{l}\text { Days } \\
\text { Group }\end{array}$ & 1 & 7 & 14 & 22 & Total & 1 & 7 & 14 & 22 & Total \\
\hline \multicolumn{11}{|l|}{ Sodium } \\
\hline Control & $\begin{array}{l}12.21 \\
\pm 0.44\end{array}$ & $\begin{array}{l}12.45 \\
\pm 0.40\end{array}$ & $\begin{array}{l}11.96^{\mathrm{A}} \\
\pm 0.31\end{array}$ & $\begin{array}{c}11.72^{\mathrm{A}} \\
\pm 0.28\end{array}$ & $\begin{array}{l}12.08^{\mathrm{A}} \\
\pm 0.18\end{array}$ & $\begin{array}{l}11.90 \\
\pm 0.37\end{array}$ & $\begin{array}{l}12.14 \\
\pm 0.42\end{array}$ & $\begin{array}{l}11.79^{\mathrm{A}} \\
\pm 0.27\end{array}$ & $\begin{array}{l}12.16^{\mathrm{A}} \\
\pm 0.25\end{array}$ & $\begin{array}{c}12.00^{\mathrm{A}} \\
\pm 0.16\end{array}$ \\
\hline T1 & $\begin{array}{l}11.91^{\mathrm{a}} \\
\pm 0.39\end{array}$ & $\begin{array}{l}13.31^{\mathrm{a}} \\
\pm 0.50\end{array}$ & $\begin{array}{c}17.50^{\mathrm{Cb}} \\
\pm 0.22\end{array}$ & $\begin{array}{c}22.47^{\mathrm{Cc}} \\
\pm 0.32\end{array}$ & $\begin{array}{c}16.30^{\mathrm{B}} \\
\pm 0.88\end{array}$ & $\begin{array}{l}11.84^{\mathrm{a}} \\
\pm 0.35\end{array}$ & $\begin{array}{l}12.80^{\mathrm{a}} \\
\pm 0.31\end{array}$ & $\begin{array}{c}15.09^{\mathrm{Cb}} \\
\pm 0.33\end{array}$ & $\begin{array}{c}19.43^{\mathrm{Cc}} \\
\pm 0.33\end{array}$ & $\begin{array}{l}14.79^{\mathrm{B}} \\
\pm 0.63\end{array}$ \\
\hline $\mathbf{T} 2$ & $\begin{array}{l}11.84^{\mathrm{a}} \\
\pm 0.35\end{array}$ & $\begin{array}{l}12.80^{\mathrm{a}} \\
\pm 0.31\end{array}$ & $\begin{array}{c}15.09^{\mathrm{Bb}} \\
\pm 0.33\end{array}$ & $\begin{array}{c}19.43^{\mathrm{Bc}} \\
\pm 0.33\end{array}$ & $\begin{array}{c}14.60^{\mathrm{Bq}} \\
\pm 0.41\end{array}$ & $\begin{array}{l}12.42^{\mathrm{a}} \\
\pm 0.48\end{array}$ & $\begin{array}{l}12.93^{\mathrm{a}} \\
\pm 0.37\end{array}$ & $\begin{array}{c}13.29^{\mathrm{Ba}} \\
\pm 0.33\end{array}$ & $\begin{array}{c}15.51^{\mathrm{Bb}} \\
\pm 0.56\end{array}$ & $\begin{array}{c}13.54^{\mathrm{Bp}} \\
\pm 0.32\end{array}$ \\
\hline \multicolumn{11}{|l|}{ Potassium } \\
\hline Control & $\begin{array}{l}76.16 \\
\pm 0.72\end{array}$ & $\begin{array}{l}77.02^{\mathrm{A}} \\
\pm 0.75\end{array}$ & $\begin{array}{l}76.74^{\mathrm{A}} \\
\pm 1.19\end{array}$ & $\begin{array}{c}77.46^{\mathrm{A}} \\
\pm 0.94\end{array}$ & $\begin{array}{c}76.85^{\mathrm{A}} \\
\pm 0.44\end{array}$ & $\begin{array}{l}79.20 \\
\pm 1.74\end{array}$ & $\begin{array}{l}77.03 \\
\pm 0.63\end{array}$ & $\begin{array}{l}79.35 \\
\pm 1.37\end{array}$ & $\begin{array}{l}76.62^{\mathrm{A}} \\
\pm 0.90\end{array}$ & $\begin{array}{l}78.05 \\
\pm 0.63\end{array}$ \\
\hline T1 & $\begin{array}{l}77.92^{\mathrm{a}} \\
\pm 0.44\end{array}$ & $\begin{array}{c}80.81^{\text {Bab }} \\
\pm 1.12\end{array}$ & $\begin{array}{c}82.22^{\text {Bab }} \\
\pm 1.90\end{array}$ & $\begin{array}{c}83.69^{\mathrm{Bb}} \\
\pm 1.48\end{array}$ & $\begin{array}{c}81.16^{\mathrm{Bq}} \\
\pm 0.77\end{array}$ & $\begin{array}{l}76.16^{\mathrm{a}} \\
\pm 0.82\end{array}$ & $\begin{array}{c}78.03^{\mathrm{ab}} \\
\pm 0.45\end{array}$ & $\begin{array}{l}78.78^{b} \\
\pm 0.55\end{array}$ & $\begin{array}{c}79.86^{\mathrm{Bb}} \\
\pm 0.47\end{array}$ & $\begin{array}{l}78.21^{\mathrm{p}} \\
\pm 0.39\end{array}$ \\
\hline $\mathbf{T} 2$ & $\begin{array}{l}76.70 \\
\pm 1.11\end{array}$ & $\begin{array}{c}77.73^{\mathrm{AB}} \\
\pm 0.82\end{array}$ & $\begin{array}{c}77.32^{\mathrm{A}} \\
\pm 0.43\end{array}$ & $\begin{array}{c}76.13^{\mathrm{A}} \\
\pm 0.55\end{array}$ & $\begin{array}{c}76.97^{\mathrm{A}} \\
\pm 0.38\end{array}$ & $\begin{array}{l}77.27 \\
\pm 0.81\end{array}$ & $\begin{array}{l}75.93 \\
\pm 0.95\end{array}$ & $\begin{array}{r}75.96 \\
\pm 1.51\end{array}$ & $\begin{array}{c}77.02^{\mathrm{A}} \\
\pm 0.75\end{array}$ & $\begin{array}{l}76.55 \\
\pm 0.50\end{array}$ \\
\hline \multicolumn{11}{|l|}{ Chloride } \\
\hline Control & $\begin{array}{c}9.94 \\
\pm 0.27\end{array}$ & $\begin{array}{l}9.84^{\mathrm{A}} \\
\pm 0.38\end{array}$ & $\begin{array}{l}10.04^{\mathrm{A}} \\
\pm 0.17\end{array}$ & $\begin{array}{l}9.80^{\mathrm{A}} \\
\pm 0.10\end{array}$ & $\begin{array}{l}9.90^{\text {Ap }} \\
\pm 0.12\end{array}$ & $\begin{array}{l}10.09 \\
\pm 0.22\end{array}$ & $\begin{array}{l}10.48 \\
\pm 0.24\end{array}$ & $\begin{array}{l}9.91^{\mathrm{A}} \\
\pm 0.18\end{array}$ & $\begin{array}{c}10.99^{\mathrm{A}} \\
\pm 0.59\end{array}$ & $\begin{array}{c}10.37^{\mathrm{Aq}} \\
\pm 0.18\end{array}$ \\
\hline T1 & $\begin{array}{l}10.19^{\mathrm{a}} \\
\pm 0.29\end{array}$ & $\begin{array}{c}11.77^{\mathrm{Bb}} \\
\pm 0.35\end{array}$ & $\begin{array}{c}14.84^{\mathrm{Cc}} \\
\pm 0.24\end{array}$ & $\begin{array}{c}18.80^{\mathrm{Cd}} \\
\pm 0.46\end{array}$ & $\begin{array}{c}13.90^{C} \\
\pm 0.70\end{array}$ & $\begin{array}{l}10.02^{\mathrm{a}} \\
\pm 0.25\end{array}$ & $\begin{array}{l}11.11^{\mathrm{a}} \\
\pm 0.32\end{array}$ & $\begin{array}{c}13.02^{\mathrm{Bb}} \\
\pm 0.42\end{array}$ & $\begin{array}{c}16.83^{\mathrm{Cc}} \\
\pm 0.37\end{array}$ & $\begin{array}{l}12.75^{\mathrm{B}} \\
\pm 0.56\end{array}$ \\
\hline $\mathbf{T 2}$ & $\begin{array}{l}9.89^{\mathrm{a}} \\
\pm 0.30\end{array}$ & $\begin{array}{c}10.41^{\mathrm{Aa}} \\
\pm 0.28\end{array}$ & $\begin{array}{c}12.04^{\mathrm{Bb}} \\
\pm 0.24\end{array}$ & $\begin{array}{c}15.22^{\mathrm{Bc}} \\
\pm 0.33\end{array}$ & $\begin{array}{l}11.89^{B} \\
\pm 0.45\end{array}$ & $\begin{array}{l}9.87^{\mathrm{a}} \\
\pm 0.23\end{array}$ & $\begin{array}{l}10.39^{\mathrm{a}} \\
\pm 0.23\end{array}$ & $\begin{array}{c}12.07^{\mathrm{Bb}} \\
\pm 0.33\end{array}$ & $\begin{array}{c}14.41^{\mathrm{Bc}} \\
\pm 0.26\end{array}$ & $\begin{array}{c}11.68^{\mathrm{AB}} \\
\pm 0.39\end{array}$ \\
\hline
\end{tabular}

$\mathrm{a}, \mathrm{b}, \mathrm{c}, \mathrm{d}$ describe significant differences within groups $(\mathrm{P}<0.05)$

A, B, C describe significant differences between groups $(\mathrm{P}<0.05)$

$\mathrm{p}, \mathrm{q}$ describe significant differences between seasons $(\mathrm{P}<0.05)$ 
Bianca (1965), McKinley et al., (1983), Thrasher et al., (1984), Mentjes and Engel Brecht (1995) and Parker et al., (2003) reported that dehydration induces natriuresis in cattle, rabbit, rats, dogs, and sheep. The increased urine sodium content due to dehydration is most probable due to a homeostatic mechanism that allows maintenance of sodium balance by the body. Hossaini-Hilali et al., (1994) observed that urinary sodium excretion increased on the second day of water deprivation. However, they found that urinary potassium excretion did not change significantly during water deprivation, but was reduced after rehydration in the lactating goats. BlairWest et al., (1972) observed that the daily urinary loss of sodium was variable without a regular trend and urinary potassium excretion was maintained at about $250 \mathrm{~m}$ equiv/day for the first 5 days, and then fell to $180 \mathrm{~m}$-equiv/day in the dehydrated sheep. In contrast, Khan et al., (1978) also reported that there was a decrease in urinary sodium concentration after the 4 day of water deprivation. Similarly, Wittenberg et al., (1986) reported that sodium and potassium excretion decreased in the water depleted goats. McKinley et al., (2000), Anastasio et al., (2001) and Parker et al., (2003) claimed that twenty four hours dehydration does not affect the daily urine output of potassium. As presented in Table 2, sodium and chloride concentrations showed increasing trend up to day 22 in both seasons in T2 group; whereas, potassium concentration showed similar trend from day 7 during winter season, but during summer concentration increased up to 7 day, then decreased. Between groups, significantly higher $(\mathrm{P}<0.05)$ sodium and chloride concentrations were observed in water deprived goats (T1) as compared to the other groups on 14 and 22 day of experiment during both seasons. Potassium concentrations were significantly higher
$(\mathrm{P}<0.05)$ in water deprived group (T1) as compared to other two groups from day 14 during summer and day 22 in winter; however, no significant variations was found between T2 and control group values. Overall summer value of sodium was higher in all groups, significant variation found in T2 group. Overall potassium concentrations was significantly higher $(\mathrm{P}<0.05)$ in $\mathrm{T} 1$ in summer. Summer chloride concentrations were higher in treatment groups; whereas, lower in control (Table 2).

\section{References}

Abdoun, K., Stumpff, F., Rabbani, I. and Martens, H. 2010. Modulation of urea transport across sheep rumen epithelium in vitro by SCFA and $\mathrm{CO}_{2}$. American Journal of PhysiologyGastrointestinal and Liver Physiology, 289: G508 -G520.

Al-Haidary, A. A., Aljumaah, R. S., Alshaikh, M. N., Abdoun, K. A., Samara, E. A., Okab, A. B. and Alfuraiji, M. M. 2012. Thermoregulatory and physiological responses of Najdi sheep exposed to environmental heat load prevailing in Saudi Arabia. Pakistan Veterinary Journal, 32(4): 515-519.

Anastasio, P., Cirillio, M., Spitali, L., Frangiosa, A., Pollastro, R. M. and De Santo, N.G. 2001.Level of hydration and renal function in healthy humans. Kidney International, 60:748-56.

Ashour, G., Benlamlih, S., Guessous, F., Rihani, $\mathrm{N}$ and Ilham, A. 2001. Adaptation of Mediterranean breeds to heat stress and water deprivation. In: (eds.), Livestock Production and Climatic Uncertainty in the Mediterranean: Proceedings of the Joint ANPA-EAAPCIHEAM-FAO Symposium. Wageningen Pers, Wageningen.

Basheir, R. A., Omer, S. A. and Mohamed, O. S. A. 2009. The effect of twenty four hours water deprivation on renal function of Sudanese Nubian goats. Journal of Computer Science and Technology, 10(1): 
1-7.

Bianca, W. J., Findlay, J. D., and Mclean, J. A. 1965.Responses of steers to water restriction. Research in Veterinary Science, 6: 38-55.

Blair-West, J. R., Brook, A. H. and Simpson, P. A. 1972. Renin responses to water restriction and rehydration. The Journal of Physiology, 226: 1-13.

Boyd, C. E. 1981. Water Quality in warm water fish ponds. Anburn University, Alabama. 359p. Craftmaster Printers, Inc. Opelika, Alabama.

Cole, N. A. 2000. Changes in postprandial plasma and extracellular and ruminal fluid volumes in wethers fed or unfed for 72 hours. Journal of Animal Sciences, 78: 216-223.

El-Nouty, F. D., Al-Haidary, A. A. and Basmaeil, S. M. 1990. Physiological responses, feed Intake, urine volume and serum osmolality of Aardi goats deprived of water during spring and summer. Asian-Australasian Journal of Animal Sciences, 3(4): 331-336.

Ghanem, A. M., Barbour, E. K., Hamadeh, S. K., Jaber, L. S. and Abi Said, M. 2008. Physiological and chemical responses in water-deprived Awassi ewes treated with vitamin C. Journal of Arid Environments, 72(3): 141-149.

Hanna, N. 2006. Physiological Changes in Water Stressed Awassi Ewes Supplemented with Vitamin C. Master Thesis. American University of Beirut; 185.

Hossaini-Hilali, J., Benlamlih, S. and Kristina D. T. 1994. Effects of dehydration, rehydration, and hyperhydration in the lactating and non-lactating black Moroccan goat. Comparative Biochemistry and Physiology, 109(4): 1017-1026.

Igbokwe, I. O. 1997. The effects of water deprivation in livestock ruminants: an overview. Nutritional Abstracts and Review (Series B), 67: 905-914.

Jaber, L. S., Habre, A., Rawda, N., Said, M. A., Barbour, E. K. and Hamadeh, S. 2004. The effect of water restriction on certain physiological parameters in Awassi sheep.
Small ruminant research, 54(1-2): 115120.

Karnib, M. 2009. The Effect of Vitamin C Administration on some Physiological Parameters in Water and Feed Restricted Dry Awassi Ewes. Master Thesis, American University of Beirut; pp.1-48.

Khan, M. S., Ghosh, P. K. and Sasidharan, T. O. 1978. Effect of acute water restriction on plasma proteins and on blood and urinary electrolytes in Barmer goats of the Rajasthan desert. The journal of agricultural science, 91(2): 395-398.

Leng, L., Szanyiova, M., Varady, S., and Boda, K. 1987. The effect of water depletion on renal excretion of urea and electrolytes in sheep. Veterinary medicine, 32: 371-8.

Madziga, I. I., Alawa, C. B. I., Lamidi, O. S. and Goska, D. Y., 2013. Assessing the nutritional status of four indigenous breeds of cattle using some blood metabolites in Nigeria. Journal of Biology, Agriculture and Healthcare, 3(2): $1-8$.

Maltz, E., Olsson, K., Glick, S. M., Fyhrquist, F., Shanikout, N., Chosniak, I and Shkolnik, A. 1984. Homeostatic responses to water deprivation or hemorrhage in lactating and non-lactating Bedouin goats. Comparative Biochemistry and Physiology, A (77A) 79-84.

Mckinley, M. J., Denton, D. A., Evred, M. D., Nelson, J. F. S., and Weisenger, R. S. 1983. Dehydration induces sodium depletion in rats, rabbits and sheep. American Journal of Physiology: Regulatory, Integrative and Comparative Physiology, 245: 287-292.

McKinley, M. J., Evered, M. D and Mathai, M. L. 2000. Renal Na excretion in dehydrated and rehydrated adrenalectomized sheep maintained with aldosterone. American Journal of Physiology: Regulatory, Integrative and Comparative Physiology, 279: 17-24.

Meintjes R. A. and Engel Brecht, H. 1995. The effect of short term dehydration on kidney function, plasma renin concentration, fecal water loss and total body water in sheep. African Journal of Science, 90(1): 27-32. 
Mengistu, U., Dahlborn, K. and Olsson, K. R. E. 2007.Mechanisms of water economy in lactating Ethiopian Somali goats during repeated cycles of intermittent watering. Animal, 1: 1009-1017.

National Research Council. 2007. Nutrient requirements of small ruminants: sheep, goats, cervids and new world camelids. Slovak Journal of Animal Science, 47(3): 149-156.

Parker, A. J, Hamlin, G. P, Coleman, C. J and Fitzpatrick L. 2003. Dehydration in stressed ruminants may be the result of a cortisol-induced diuresis. Journal of Animal Science, 81: 512-519.

Qinisa, M. M. 2010. Effect of water restriction on aspects of digestion in Boer and Tswana goats offered Eragrostisteff and Lucerne Hay, $\mathrm{PhD}$ Thesis submitted to University of Pretoria.

Rajkhowa, S. and Hazarika, G. C. 2000. Clinical-Biochemical studies on the effect of water deprivation on goats under hot climatic conditions. Indian Veterinary Journal, 77: 856-858.

Rathwa, S. D., Vasava, A. A., Pathan, M. M., Madhira, S. P., Patel, Y. G. and Pande, A. M. 2017. Effect of season on physiological, biochemical, hormonal, and oxidative stress parameters of indigenous sheep. Veterinary World, 10: 650-654.

Samara, E. M., Abdoun, K. A., Okab, A. B. and Al-Haidary, A. A. 2012. A comparative thermo-physiological study on waterdeprived goats and camels. Journal of Applied Animal Research, 40(4); 316-322.

Silanikove, N. 1992: Effects of water scarcity and hot environments on appetite and digestion in ruminants: a review. Livestock Production Science, 30: 175194.

Snedecor, G. W. and Cochran, W. G. 2004. Statistical Methods, 8 Edn., Oxford and IBH Pub. Co., Kolkata.

Thrasher, T. N., Wade, C. E., Keil, L. C. and Ramsay, D. J. 1984.Water balance and aldesterons during dehydration in the dog. American Journal of PhysiologyRegulatory, Integrative and Comparative Physiology, 247: 76-83.

Varady, K. A., Roohk, D. J., Loe, Y. C., McEvoy-Hein, B. K, and Hellerstein, M. K. 2007. Effects of modified alternate-day fasting regimens on adipocyte size, triglyceride metabolism, and plasma adiponectin levels in mice. Journal of Lipid Research, 48: 2212-2219.

Wittenberg, C., Choshniak, I., Shkolnik, A., Thurau, K. and Rosenfeld, J. 1986. Effect of dehydration and rapid rehydration on renal function and on plasma renin and aldosterone levels in the black Bedouin goat. Pflugers Archiv European Journal of Physiology, 406(4): 405-408.

Wojtas, K., Cwynar, P. and Kolacz, R. 2014. Effect of thermal stress on physiological and blood parameters in merino sheep. Bulletin Veterinary Institute and Pulaway, 58: 283-288.

Zetingrao, G. N. 2003. Effects of water deprivation in osmanabadi goats of Marathwada region. Ph D. Thesis submitted to Maharashtra Animal \& Fishery Sciences University, Nagpur.

\section{How to cite this article:}

Sumeet Kour, Jonali Devi, Kamal Sarma, Dibyendu Chakraborty, Aafreen Wahid Ganai, Pallavi Khajuria and Sourab Dua. 2019. Effect of Vitamin C Supplementation on Electrolyte Concentrations in Blood and Urine of Water Deprived Goats. Int.J.Curr.Microbiol.App.Sci. 8(12): 2689-2697. doi: https://doi.org/10.20546/ijcmas.2019.812.315 